\title{
An open architecture for the massively parallel emulation of the Drosophila brain on multiple GPUs
}

Lev E Givon, Aurel A Lazar

From Twenty First Annual Computational Neuroscience Meeting: CNS*2012

Decatur, GA, USA. 21-26 July 2012

The fruit fly Drosophila melanogaster is an exceedingly useful model organism for studying the causal links between neural circuits and behavior due to the numerical tractability of its brain and its powerful neurogenetic toolkit. Recent progress made in identifying the connectome of the fruit fly $[1,2]$ and in characterizing the input and output functions of its sensory neural circuits [3] raise the possibility of creating and emulating a functional

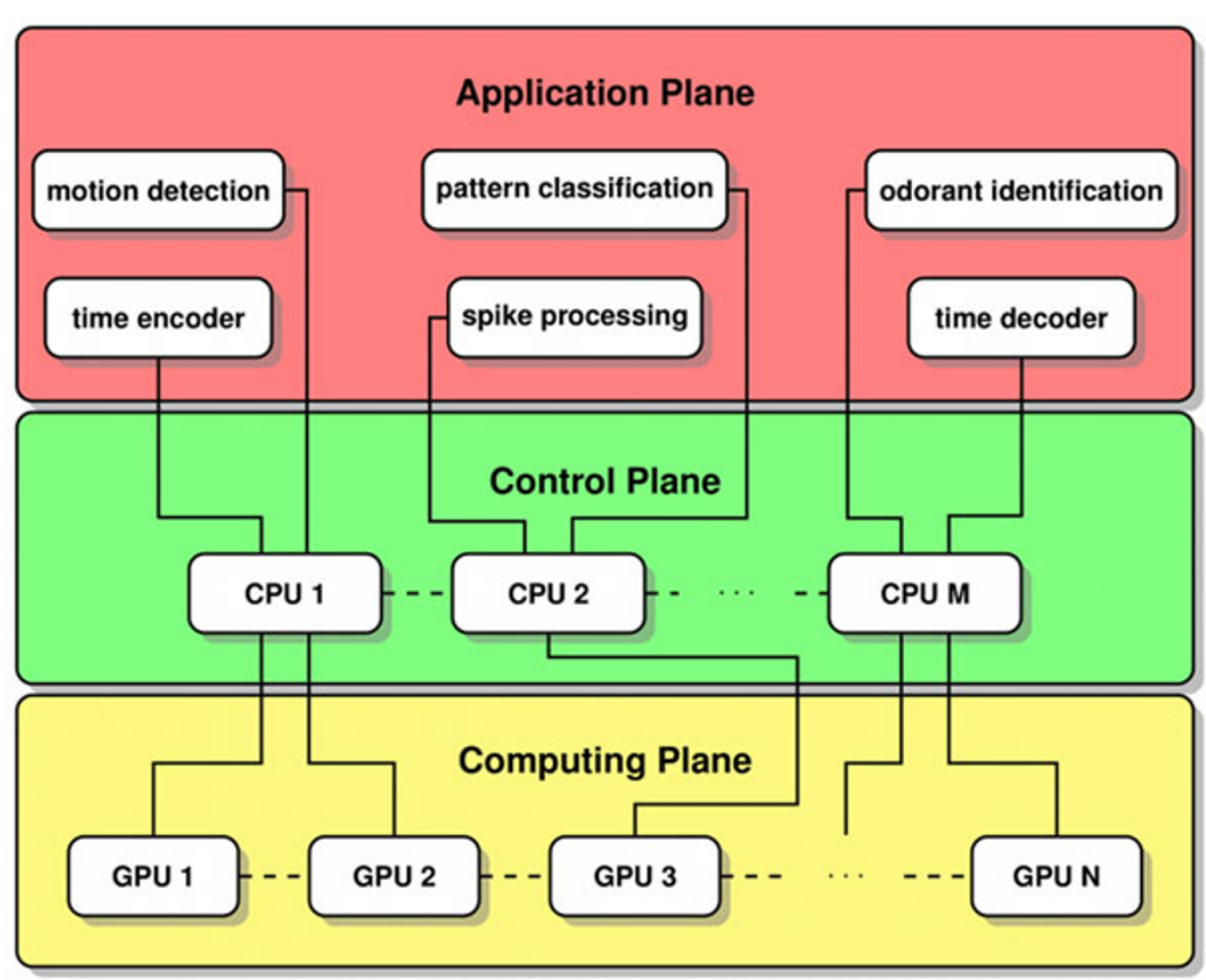

Figure 1 Neurokernel architecture

* Correspondence: aurel@ee.columbia.edu

Department of Electrical Engineering, Columbia University, New York, NY 10027, USA 
model of the entire fly brain using the increasingly powerful commodity parallel computing technology available to computational neuroscientists. To this end, we have developed an open software architecture for emulating neural circuit modules in the fly brain and their responses to recorded or simulated input stimuli on multiple Graphics Processing Units (GPUs). A key feature of this architecture is its support for integrating instances of different neural circuit models developed by independent researchers by requiring that the models' implementations provide interoperable interfaces that adhere to the specification prescribed by the architecture.

We refer to the architecture as a Neurokernel because it provides object classes essential to the emulation of the entire fruit fly brain that are analogous to those provided by an operating system kernel: (1) it serves as an extended machine that provides access to neural circuit primitives needed to construct and interconnect models of neural circuit modules in the fly brain; and (2) it serves as a resource allocator that scalably and transparently assigns GPU resources to emulated neural circuit models without manual specification by the researcher [5]. In order to provide these features, the Neurokernel architecture comprises several planes of abstraction that separate its application, control, and computing aspects (Fig. 1). Models of brain function implemented using the architecture use the application plane's API to access neural circuit primitives without directly specifying which GPU resources to use. The architecture's control plane automatically partitions and maps circuits to available GPU resources, and manages communication between multiple GPUs hosted locally or remotely. Storage methods used to efficiently represent large networks of neurons and synapses with feedback connections in GPU memory and numerical methods used to update neuron and synapse states are implemented in the computing plane.

We implemented key elements of the Neurokernel software using the Python programming language and the PyCUDA interface to NVIDIA's CUDA GPU programming environment [4] to avail ourselves of the increasingly powerful ecosystem of scientific computing Python software and make the architecture accessible to other researchers in the neuroscience community.

Published: 16 July 2012

\section{References}

1. Chiang AS, Lin CY, Chuang CC, Chang HM, Hsieh CH, Yeh CW, Shih CT, Wu JJ, Wang GT, Chen YC: Three-dimensional reconstruction of brainwide wiring networks in Drosophila at single-cell resolution. Curr Biol 2011, 21(1):1-11.

2. Chklovskii DB, Vitaladevuni S, Scheffer LK: Semi-automated reconstruction of neural circuits using electron microscopy. Curr Opin Neurobiol 2010, 20(5):667-675

3. Kim AJ, Lazar AA, Slutskiy YB: System identification of Drosophila olfactory sensory neurons. J Comput Neurosci 2011, 30(1):143-161.
4. Klöckner A, Pinto N, Lee Y, Catanzaro B, Ivanov P, Fasih A: PyCUDA and PyOpenCL: a scripting-based approach to GPU run-time code generation. Parallel Comput 2012, 38(3):157-174.

5. Lazar AA: Programming telecommunication networks. IEEE Network 1997. 11(5):8-18.

doi:10.1186/1471-2202-13-S1-P99

Cite this article as: Givon and Lazar: An open architecture for the massively parallel emulation of the Drosophila brain on multiple GPUs. BMC Neuroscience 2012 13(Suppl 1):P99.

\section{Submit your next manuscript to BioMed Central} and take full advantage of:

- Convenient online submission

- Thorough peer review

- No space constraints or color figure charges

- Immediate publication on acceptance

- Inclusion in PubMed, CAS, Scopus and Google Scholar

- Research which is freely available for redistribution

Submit your manuscript at www.biomedcentral.com/submit
C Biomed Central 